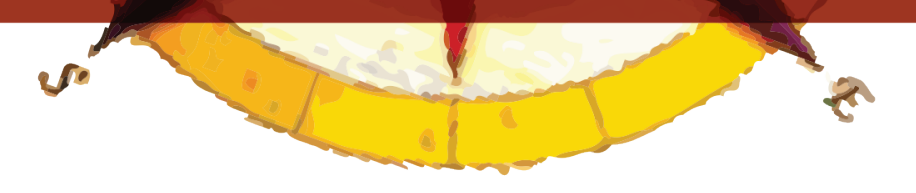

\title{
GEOTECNÓSFERA. TECNOLOGÍAS DE LA INFORMACIÓN GEOGRÁFICA EN EL CONTEXTO GLOBAL DEL SISTEMA MUNDO
}

\author{
Gustavo D. Buzai ${ }^{1}$ \\ Ernest Ruiz ${ }^{2}$
}

\section{RESUMEN}

El presente trabajo analiza los grandes componentes del sistema tierra y sistema mundo centrando su atención en la esfera tecnológica como Tecnósfera. Particularmente se analiza de qué manera su composición está cada vez más influida por tecnologías en las cuales la dimensión espacial es central, motivo por el cual es posible definir una Geotecnósfera de alcance planetario.

Con origen en la actividad científica, las Tecnologías de la Información Geográfica (TIG), comienzan a tener un uso social generalizado, por lo tanto, el nuevo contexto se enfoca desde diversas escalas. Desde el mundo (globalización de los datos a través de la red) hasta el individuo (voluntarios geográficos), el contexto actual presenta posibilidades y retos de gran importancia para lograr un mundo con vínculos en los que se privilegia el verdadero conocimiento.

\section{Palabras clave: Geotecnósfera / TIG / SIG / Geoinformática / Neogeografía}

\section{RESUMO}

O presente trabalho analisa os grandes componentes do Sistema Terra e Sistema Mundo, dando maior atenção à esfera tecnológica como Tecnosfera. Particularmente analisa-se de que maneira a sua composição está cada vez mais influenciada por tecnologias para as quais a dimensão espacial é central, de modo que é possível definir uma Geotecnosfera de alcance planetário.

Com origem na atividade científica, as Tecnologias da Informação Geográfica (TIG) começam a ter um uso social generalizado, portanto, o novo contexto é enfocado a partir de diversas escalas. Desde o mundo (globalização do dados através da rede) até o indivíduo (voluntários geográficos), o contexto atual apresenta possibilidades e desafios de grande importância na construção de um mundo com vínculos nos quais o verdadeiro conhecimento é privilegiado.

$1 \quad$ Universidad Nacional de Luján, Departamento de Ciencias Sociales

2 Universidad de Barcelona, Departamento de Geografía Humana 
Palavras-chave: Geotecnosfera / TIG / SIG / Geoinformática / Neogeografia

\begin{abstract}
This article analyzes the main components of the earth system and the world system, focusing its attention on the technological sphere as Technosphere. In particular it analyzes how the technosphere is influenced by technologies with a special interest on the spatial dimension and how, as result of this influence, it is possible to define a global Geotechnosphere.

With its origins in the scientific activity, Geographical Information Technologies (GIT), are beginning to be widely-used by society, and so, the new context is observed from diverse scales. From the world (globalization of data through the internet) to the individual (geographic volunteers), the actual context shows possibilities and challenges of great importance in order to assure a linkedworld in which true knowledge is granted.
\end{abstract}

Keywords: Geotechnosphere / GIT/ GIS/ Geoinformatics /Neogeography

\title{
INTRODUCCIÓN
}

Las Tecnologías de la Información Geográfica (TIG) han tenido un desarrollo notable en el ámbito de la Geoinformática, un contexto científico-tecnológico que posibilitó la convergencia de diferentes componentes a partir del núcleo formado por los Sistemas de Información Geográfica (SIG) durante su medio siglo de existencia (1964-2014).

Aspectos tradicionales en los estudios geográficos, como lo es la relación de las sociedades con su medio, resultan posibles cuando se centran en el uso de las tecnologías que lo mediatizan. Junto a la Geósfera y Biósfera el hombre ha generado su propio medio contextual a través de la Tecnosfera, un mundo de artefactos tecnológicos que le brinda posibilidades para actuar en el planeta.

En las últimas décadas la tecnosfera se está poblando de tecnología geográfica, elementos que se centran en la localización, valorizan notablemente las distribuciones espaciales y, con ello, favorecen el desarrollo de la inteligencia espacial de la sociedad. Las TIG han generado la Geotecnósfera, una esfera basada en las tecnologías digitales que permiten interactuar y conocer cada vez más la realidad geográfica que nos rodea.

De esta manera la Geotecnósfera contribuye al pensamiento y se transforma en elemento concreto fundamental para la conformación de la Noósfera, como esfera de conocimiento e inteligencia planetaria. Cada una en diferentes escalas proporciona la posibilidad de tener un mundo más conectado, más instantáneo y cercano, con datos geográficos para entender la dinámica geográfica que interactúa en la vida cotidiana como base empírica.

Partiendo de estas perspectivas contextuales, se constata la actual globalización apoyada por las tecnologías digitales en red (Internet) y las posibilidades de comprender estas situaciones a través de la teoría sistémica, en la cual la realidad como sistema complejo brinda elementos demarcatorios que permiten abordar con suficiencia diferentes escalas de análisis. 
En este texto se analiza la relación entre el análisis espacial y el análisis geográfico con base en sus contenidos tecnológico, científico y nivel de escala, y también, aspectos conceptuales y evolutivos de la Geoinformática. En ambos casos aparece la Neogeografía y la utilización de datos geográficos masivos como los componentes concretos que permiten claras ampliaciones actuales.

La Geoinformática, con un comienzo centrado en un desarrollo científico-tecnológico de alcance académico actualmente se expande de manera notable.

En este contexto, y a mayor nivel de detalle, aparece la población a través del uso de estas tecnologías y la conformación de un voluntariado geográfico de alcance mundial. Usuarios geotecnológicos y generadores de información geográfica que se hace disponible en red con alcance global.

La humanidad crea, desarrolla y es impactada por la Geotecnósfera. El trabajo realizado tiene por objetivo analizarla en sus diferentes dimensiones.

\section{BASE EMPÍRICA Y TECNÓSFERA}

La actual investigación aplicada en Geografía se apoya decididamente en el uso de las TIG. De entre todas estas tecnologías, los SIG han producido una importante revolución tecnológica disciplinar y, al mismo tiempo, han favorecido una innegable revolución intelectual. La revolución tecnológica se encuentra estrechamente ligada a los métodos y técnicas que se han estandarizado digitalmente con la finalidad de realizar con suficiencia el tratamiento de datos referenciados espacialmente. La revolución intelectual se relaciona directamente con la forma en la que puede representarse y ser pensada la realidad, es decir, la materialidad espacial de la base empírica del planeta.

Esta base empírica se presenta como un paisaje global formado por cuatro esferas con diferentes ritmos de evolución: la geósfera, de componentes abióticos, formada por la atmósfera, litosfera e hidrosfera, la biósfera, de componentes bióticos, formada por los seres vivos del planeta, la tecnosfera como esfera tecnológica, medio de la vida humana y la noósfera, de componente conceptual y simbólico, formada por la sociedad humana en tanto esfera de conocimientos.

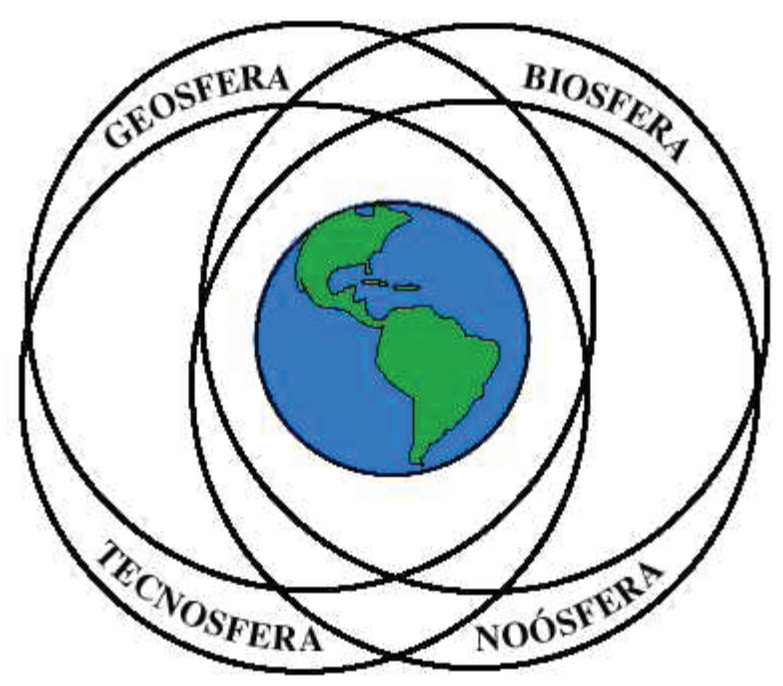

Figura 1. Esferas del planeta Tierra.

Fuente: Los autores en base a Buzai (2013a) 
La tradicional definición de la Geografía, como ciencia que estudia la relación de la sociedad con su medio, tiene su principal sustento en la tecnosfera, ya que esta esfera es la que contiene los instrumentos construidos por el hombre que permiten este vínculo y, al mismo tiempo, posibilita brindar diferentes respuestas al determinismo que aún impone la geografía sobre la historia.

Desde un punto de vista amplio la tecnoesfera abarca todo sistema organizativo humano que genera un sistema mundo cada vez más artificial, y actualmente, cada vez más digital. La tecnosfera tiene un fundamento teórico generado en la noósfera e impacta con fuerza creciente sobre la geosfera y biosfera.

La mayoría de los instrumentos tecnológicos que incluye la tecnosfera se ubican sobre la superficie terrestre, pero ésta expande su alcance permanentemente avanzando hacia el espacio exterior a través de naves espaciales, principalmente satélites artificiales de múltiples propósitos, algunos (la mayoría) en órbita terrestre y otros transitando hacia el exterior del sistema solar.

Actualmente, la tecnoesfera constituye la esfera de mayor dinamismo, debido a que crece de manera exponencial y se presenta como base material en el desarrollo de la inteligencia planetaria en red en la definitiva globalización de la humanidad, estructurándose actualmente a través del ciberespacio.

La noósfera, por lo tanto, se encuentra formada por las interacciones surgidas a través de la inteligencia humana en las redes globales que entran en relación con todas las esferas del planeta Tierra. Supera a la biosfera al realizar una transformación empírica de los elementos terrestres controlando la naturaleza (Vernadsky) y avanza en la formación de una conciencia planetaria (Teilhard de Chardin) aún un tanto lejana.

En este sentido, queda claro que aunque el hombre pertenece inicialmente a la esfera biótica, su separación de la biósfera se realiza principalmente por su componente cultural y simbólico, ya que mientras la mayoría de los seres vivos tienen como finalidad sobrevivir en el mundo, el hombre, como especie, es el único que intenta comprenderlo y mediante este intento ha creado un cuerpo de ideas y conceptos que ha dado lugar al desarrollo de la ciencia.

La ciencia puede definirse como un conocimiento racional, sistemático, exacto, verificable, y que por consiguiente, puede ser falible (Bunge, 1981).

Es racional porque es producto de la razón humana, proviene de la mente de los individuos. Se rige, sobre todo, por aspectos lógicos e intenta apartarse de las cuestiones emocionales y valorativas en la construcción de conocimientos. Si las cuestiones valorativas se producen, principalmente en el caso de las Ciencias Sociales, éstas deberán quedar explícitas.

Es sistemática porque no es un conocimiento que se produzca de manera aleatoria, sino que existen una serie de procedimientos estandarizados para su formulación. Tiende a ser exacto porque cada aproximación a la realidad busca ampliar cada vez más el grado de correspondencia entre los enunciados observacionales y la base empírica del mundo real. Y, finalmente, puede ser verificable a través de contrastar las hipótesis formuladas con el fin de aproximarnos a la verdad (verdad entendida como adecuación), la cual en la ciencia nunca puede tomarse como definitiva, sino que es provisoria. 
Cabe destacar, que en las investigaciones de Geografía Aplicada con diferentes objetivos, tales como los exploratorios, los descriptivos, los clasificatorios o los explicativos (Buzai, Baxendale y Cruz, 2009), los SIG se presentan como importantes instrumentos en la generación de hipótesis y sus posibles respuestas.

La racionalidad humana, la ciencia y la tecnología actual son los principales recursos de los que se dispone para comprender el mundo. La Geografía como ciencia aplicada los utiliza para generar elementos que permitan acceder a soluciones de carácter socioespacial.

\section{AVALANCHA DE DATOS EN LA TERCERA GLOBALIZACIÓN}

La base empírica del planeta Tierra, a través de sus esferas componentes, es una fuente de estímulos permanente. Muchos de ellos son captados de manera directa con nuestras posibilidades cognitivas e indirectamente con instrumentos cada vez más sofisticados. A este respecto algunos datos resultan sorprendentes: cuando en el año 1967 se puso en órbita por primera vez el satélite artificial LANDSAT, utilizado para la exploración de los recursos naturales y finalizó su primera circunferencia orbital sobre el planeta Tierra, obtuvo un volumen de datos equivalente al que los geógrafos habían acumulado hasta el siglo XV, y en la segunda órbita elevó el caudal de información hasta alcanzar el equivalente al obtenido a lo largo de todo el siglo XIX (Stotman, 1999).

Podemos afirmar, sin temor a equivocarnos, que esta avalancha de datos sobrepasó inmediatamente muchas de nuestras capacidades técnicas. Pero no así las capacidades de la racionalidad humana, que puede afrontar situaciones como la descrita mediante la integración y la construcción de conceptos genéricos, captando la unidad en la diversidad de manera no contradictoria (Sagan, 1995; Rand, 2011).

Observando desde una perspectiva global podemos considerar que el uso de los satélites artificiales también generó una notable revolución intelectual. Porque una cosa era saber que vivíamos en el globo terráqueo y, otra muy distinta fue contemplar nuestro planeta como esa pequeña esfera flotando en el espacio, lo cual generó un impacto conceptual y ético mayor a lo que originalmente se hubiera imaginado (Gould, 1987; Buzai, 2008).

Este impacto genera una revisión conceptual de la tradicional postura del etnocentrismo, es decir, mirar el mundo en base a los parámetros de la propia cultura, para avanzar hacia una perspectiva de mayor amplitud. La visión del Planeta Tierra desde el espacio exterior nos hace tomar conciencia del ínfimo lugar que ocupamos en el universo y que todos nos encontramos en un pequeño hogar planetario (Sagan, 1980), donde las diferencias entre el yo y el otro carecen de sentido.

Esta perspectiva planetaria como imagen en movimiento de una esfera brillante, azul por los océanos, marrón por los continentes y blanca por los hielos y las nubes, muestra desde un punto de vista empírico la mayor integración del sistema físico-natural, el sistema Tierra. Sobre este sistema existe la dimensión socio-espacial que se integra desde la escala global hacia todas las escalas posibles a través de flujos tangibles e intangibles que materializan las infinitas relaciones del sistema Mundo. Ambos sistemas específicos cuentan con vínculos dinámicos analizados por Dollfus (1992). 
Cuando existen datos de masiva presencia que fluyen por todo el sistema mundo se hace referencia al proceso de globalización. Consideramos que existen tres claras globalizaciones:

Primera globalización (material): Los primeros datos que se diseminaron por el globo fueron los que contiene el ADN de la especie humana. El primer proceso de globalización sucedió, pues, cuando fuimos la primera especie animal en poblar la totalidad de ecosistemas terrestres conectados (Lévy, 2012; Picq, 2012). Este proceso fue un largo camino que comenzó hace 7 millones de años y finalizó hace 20 mil años.

Segunda globalización (conceptual): La segunda globalización está relacionada con el pensamiento, con el momento histórico en el cual la humanidad toma conciencia que está viviendo sobre un globo que flota en el espacio. En ese momento, se advierte que existen unas antípodas y que en ellas hay gente que "vive cabeza abajo". Este segundo proceso de globalización se produjo, de esta manera, en el período de la revolución científica a partir del siglo XV, período que transcurre desde Copérnico a Newton y que ha sido definido inicialmente por Alexandre Koyré, siendo Boido (1996) quién lo estructura centrándolo en el trabajo de Galileo Galilei.

Tercera globalización (digital): La actual globalización está relacionada con la circulación de datos en el ciberespacio. Desde la cibergeografía hemos realizado diferentes cibermapas centrados en Buenos Aires a partir de los cuales es posible representarse la circulación global de información con una distribución espacial en la que queda clara una fuerte dependencia latinoamericana hacia los países centrales, principalmente Estados Unidos que ocupa más del 80\% de la frontera ciberespacial argentina (Buzai, 2013b; Focás, 2013).

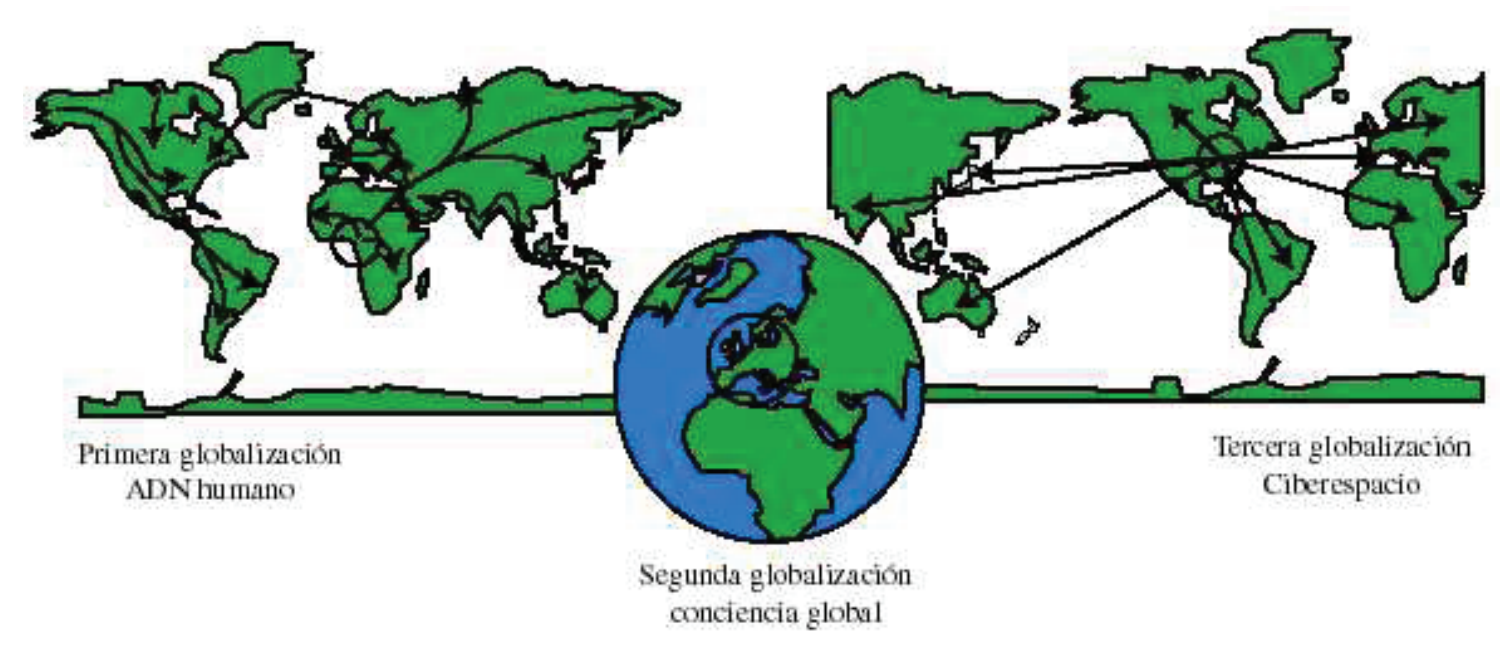

Figura 2. Globalizaciones

Fuente: Los autores, en base a información obtenida en http://es.wikipedia.org/wiki/ Evoluci\%C3\%B3n humana y Buzai (2013b)

A partir de la perspectiva planetaria, los estudios que abordan el cambio climático global, las relaciones económicas y políticas internacionales, y la lucha por el control de los espacios lineales de flujos, incluyendo la circulación ciberespacial, han tomado un importante protagonismo en el mundo actual. Corresponden a configuraciones espaciales que nos muestran las más amplias vinculaciones dentro del espacio relacional mundial y de allí hacia diferentes escalas de análisis hasta 
llegar a los espacios locales como sitios.

La Geografía, como ciencia, contempla una base empírica de gran amplitud. Todo lo que estudia se encuentra localizado en el espacio geográfico y la investigación debe definirse en una escala que se encuentra entre los 510 millones de kilómetros cuadrados del planeta y el metro cuadrado que cada uno se encuentra ocupando en este momento.

\section{LA REALIDAD GEOGRÁFICA COMO SISTEMA}

Las escalas globales nos llevan a pensar en el abordaje de la realidad como totalidad y de esta manera como sistema. En los estudios de Geografía Aplicada, la intención es utilizar los conocimientos teóricos y, a partir de ellos, avanzar en la resolución de problemáticas socio-espaciales. Es en este sentido que se plantea la cuestión acerca de la manera en que puede ser captada la realidad.

En esta línea de pensamiento, un desarrollo conceptual que avanza sobre las características centrales presentadas por la teoría general de los sistemas basada en sistemas generales (von Bertalanffy, 1968), se puede encontrar en la teoría de los sistemas complejos que se asienta en aspectos constructivistas de la epistemología genética para el trabajo científico disciplinario e interdisciplinario (García, 2006).

La teoría de los sistemas complejos ha demostrado muy buena capacidad para el estudio de la realidad en general. En concreto se ha podido comprobar su aptitud para el estudio de la realidad socio-espacial empírica de la Geografía y, también, para conceptualizar aspectos específicos correspondientes a la construcción de conocimientos en su capacidad de alcance epistemológico (Piaget y García, 1983; García, 1997).

Las TIG, en el contexto global del sistema mundo, incorporan la dimensión espacial a la tecnosfera en la que se manifiesta un creciente peso relativo frente a otras tecnologías. La teoría de los sistemas complejos permite justificar un creciente nivel de aplicación de las TIG en relación a la ampliación de la inteligencia espacial por parte de la sociedad.

\section{PERSPECTIVA EMPÍRICA}

El planeta Tierra (el Sistema Tierra) junto a las mundializaciones históricas (el Sistema Mundo) representan la realidad empírica de mayor extensión para los estudios geográficos y provee el dominio material de la Geografía como ciencia. Este abordaje corresponde al de una ciencia empírica, la que estudia hechos geográficos concretos a través del análisis espacial e intenta actuar sobre ellos.

Considerar la actividad científica, y con ello a la Geografía, como ciencia empírica no significa avalar el empirismo como actividad objetiva que capta puramente los hechos de la realidad a través de la experiencia pura. Sin embargo la razón humana se presenta como la principal fuente del conocimiento científico y minimiza los grados de arbitrariedad en el momento de realizar generalizaciones no contradictorias avanzando en la construcción de conocimientos. Esto resulta posible porque la realidad existe de forma independiente del observador (Rand, 2011) y es por eso que es posible verificar claramente que el análisis de la realidad puede orientarse hacia una función cognitiva y una función de manipulación (Soros, 2010). 
Desde una postura constructivista los sistemas no están definidos y son definibles. La construcción sistémica en cualquier escala y extensión se realiza a través de los datos como estímulos generados por la realidad, los observables como datos interpretados por el observador, y los hechos formados por la relación entre observables.

Una cuestión central a ser resaltada en esta instancia es que a través de esta perspectiva se aborda la realidad como una estructura perteneciente a una totalidad estratificada, es decir, una realidad formada por niveles de organización semi-autónomos, en los que rigen dinámicas específicas para cada uno de ellos, aunque interactúan entre si desde un punto de vista multiescalar.

Esta consideración posibilita contar con un marco conceptual general que sustenta la estabilidad de determinadas teorías en niveles específicos y la no-invalidación de ellas a través de consideraciones que pertenecen claramente a otros niveles de análisis. Consideramos que esto sería una perspectiva en tercera dimensión de la metodología de los programas de investigación en el marco del falsacionismo sofisticado propuesto por Lakatos (1977).

Cuando se estudian sistemas generales es posible encontrar comportamientos similares en diferentes escalas. Tanto en la distribución espacial de una galaxia, como en la de una ciudad o en la de un conjunto de neuronas, es posible observar una estructura geométrica con rasgos similares que pueden ser comprendidos a través de conceptos surgidos de la geometría fractal (Buzai y Cacace, 2013).

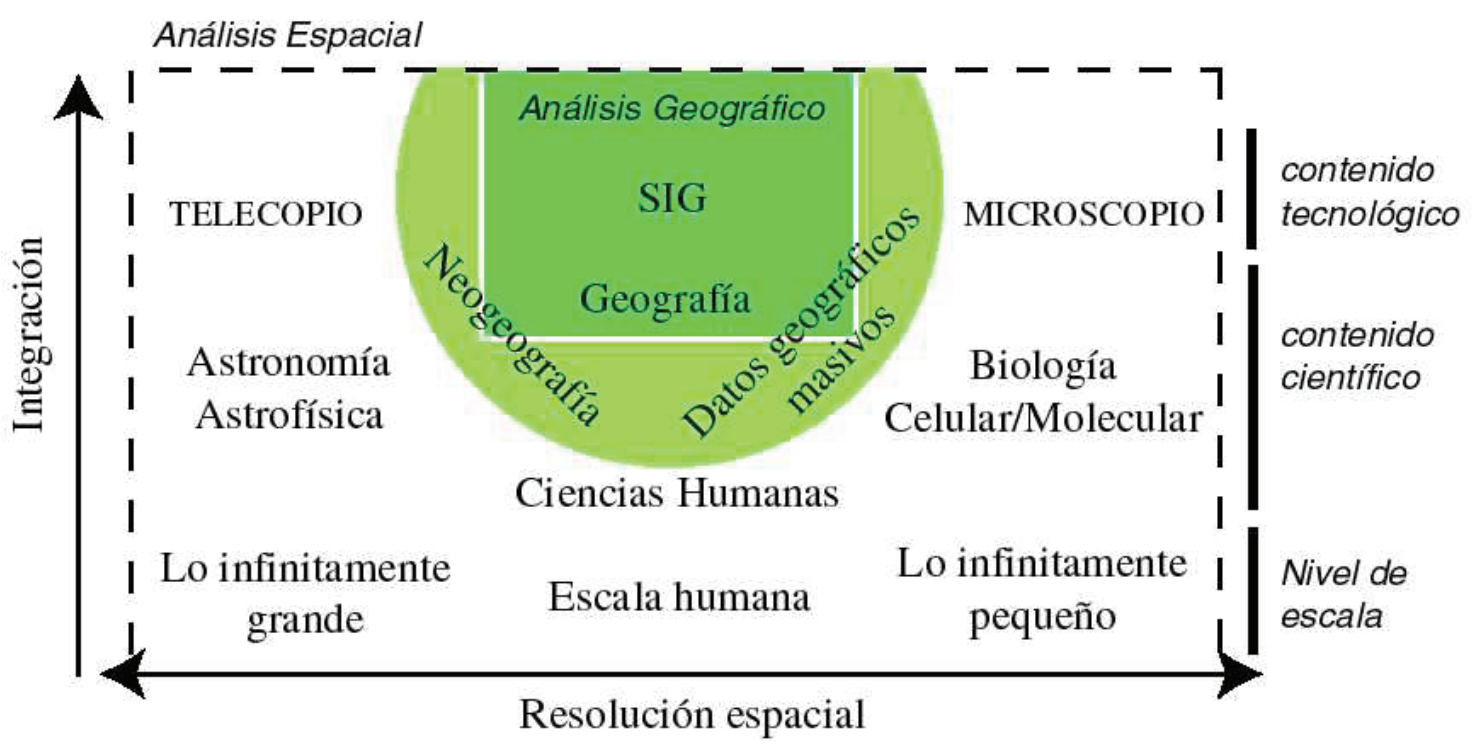

Figura 3. Análisis espacial y análisis geográfico

Fuente: Los autores en base a Buzai y Baxendale (2011)

Cuando se estudian sistemas complejos es posible utilizar teorías diferentes y específicas para cada escala. Entre lo infinitamente grande y lo infinitamente pequeño se encuentra la escala humana, que puede considerarse un espacio infinitamente complejo, en el sentido de complicado (de Rosnay, 1977). La Geografía es una de las ciencias más interesantes y dinámicas ya que debe afrontar las complejidades físico-naturales, las complejidades humanas, y las complejidades de la relación entre la sociedad y su medio. Para cada escala es posible utilizar tecnologías específicas para el análisis de 
cada una de ellas. Así pues, mientras para lo infinitamente grande puede hacerse uso de telescopios y para lo infinitamente pequeño de microscopios, los SIG son la tecnología específica para estudiar la dimensión espacial de la escala humana.

Existe un avance continuo que sigue la evolución GISystem, GIScience, GISociety (Burrough y McDonnell, 1998). La Geotecnosfera, a través de la Neogeografía y el uso de datos geográficos masivos amplía las posibilidades del análisis geográfico a otras escalas, trasladando el análisis geográfico de la Geografía Global a ámbitos extracientíficos a partir de un uso social generalizado.

\section{EVOLUCIÓN GEOINFORMÁTICA}

El término Geografía Automatizada hace referencia al proceso por el cual, a partir de iniciada la década de 1980, se incorporan al análisis espacial nuevas posibilidades de resolución a partir de las tecnologías digitales.

Estas tecnologías digitales presentan una gran variedad de posibilidades de aplicación y los Sistemas de Información Geográfica, como tecnología de integración, se han convertido definitivamente en el principal medio para realizar un análisis socioespacial con el fin de proveer caminos de solución a las problemáticas concretas que demandan una efectiva gestión y planificación territorial.

La valorización generalizada de estas aplicaciones ha sido muy importante y su prestigio ha crecido progresiva y simultáneamente a la incorporación conceptual de las variables de localización $(x, y)$, de atributos $(z)$ y de tiempo $(t)$ en estudios interdisciplinarios. En la práctica, la totalidad de dimensiones se consideran imprescindibles para un análisis realizado lo más completamente posible del mundo real.

La transformación del mundo real en un modelo digital con posibilidades de ser trabajado mediante procedimientos computacionales, exige una serie de complejas operaciones conceptuales que finalizan al nivel de byte. Mediante estas transformaciones, que comportan procesos de fragmentación y estandarización de la información espacial, todo objeto geográfico puede definirse digitalmente a través de una geometría particular (punto, línea, polígono, raster o $x$-tree), una localización precisa en el espacio absoluto ( $x-y$ o geográficas), una serie de atributos (campos de informaciónvariables o capas temáticas-layers) y su existencia en un momento histórico (instante de realización de las mediciones).

La concreción de estos aspectos mediante medios computacionales se logra a través de la generación de bases de datos alfanuméricas y bases de datos gráficas.

Las primeras se encuentran asociadas al almacenamiento de datos alfanuméricos que representan los atributos de cada entidad ubicada en el espacio geográfico, y los software que se utilizan para su tratamiento son los Editores de Textos (EDT), Administradores de Bases de Datos (ABD), Planillas de Cálculo (PLC), Programas de Análisis Estadístico (PAE) y Sistemas de Posicionamiento Global (GPS). ${ }^{3}$

Las segundas se encuentran asociadas al almacenamiento de los aspectos geométricos, y los soft-

Se conserva la sigla en inglés, GPS (Global Positioning System). 
ware que se utilizan para su tratamiento son los programas de Diseño Asistido por Computadora (CAD), Mapeo Asistido por Computadora (CAM), Gestión de Infraestructura (AM-FM), Sistema de Información de Tierras (LIS), Procesamiento Digital de Imágenes (PDI) y Modelado Numérico de Terreno (MNT). ${ }^{4}$

Ambos grupos de tecnologías han experimentado un continuo proceso de convergencia a través de la creciente compatibilidad de software durante más de dos décadas (1964-1990) para, posteriormente, registrar un avance hacia la plena circulación de resultados en los siguientes dos decenios (1990-2013).

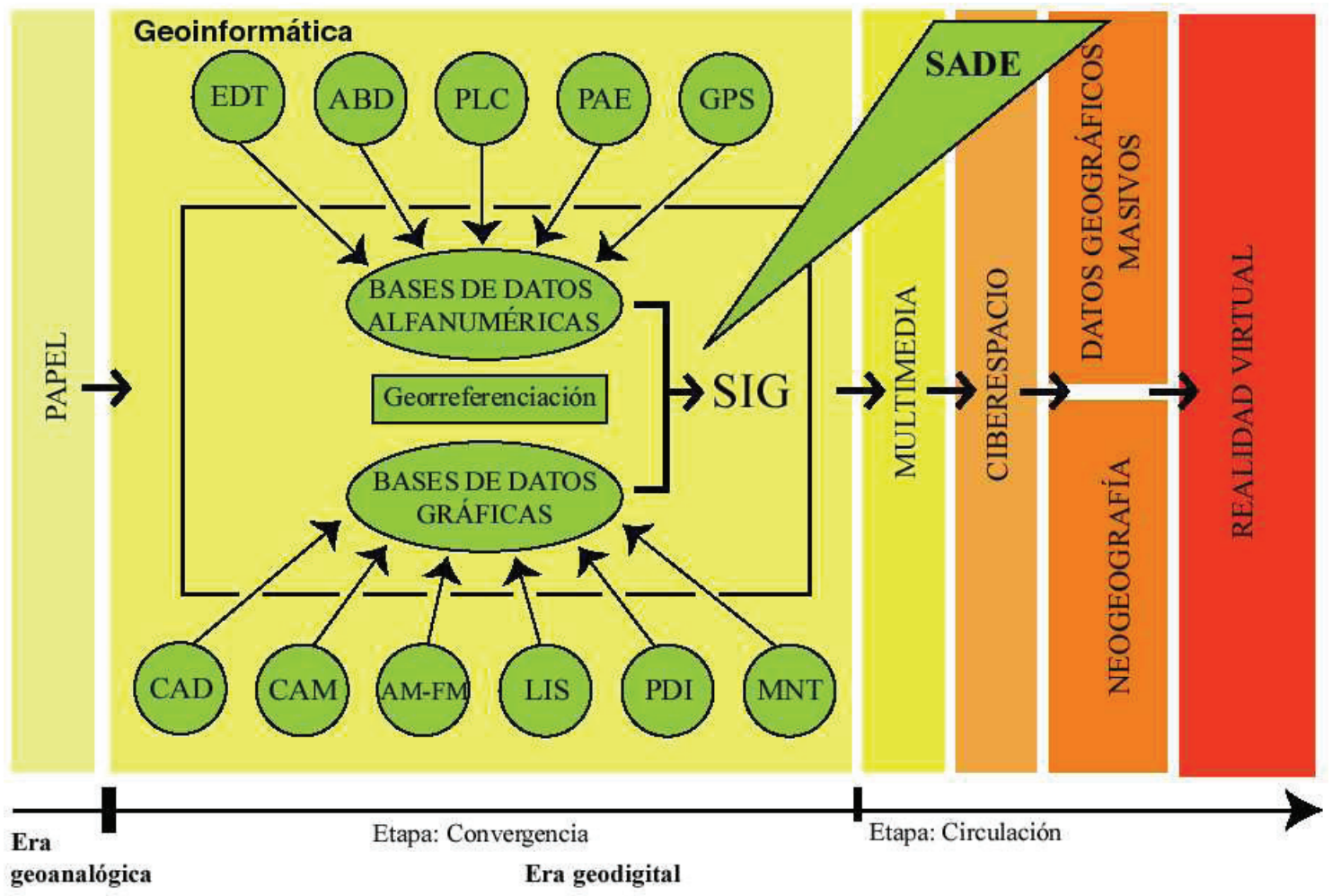

Figura 4. Relaciones Geoinformáticas

Fuente: Los autores en base a Buzai y Baxendale (2011)

Cuando se combinan las bases de datos alfanuméricas y gráficas y se referencian espacialmente a un sistema de coordenadas geográficas (Georreferenciación), surge el concepto de Sistemas de Información Geográfica (SIG). En la figura 4 puede verse de forma gráfica que si salimos de este núcleo hacia sus bordes encontramos diferentes tipos de software en una estructura convergente y que en su totalidad forman el campo de la Geoinformática.

De este modo, la Geoinformática se convierte en un campo de gran amplitud en el cual se pueden incluir todo tipo de software de aplicación y del cual la tecnología SIG es su núcleo al tomar el lugar central. Esto es posible porque la Geoinformática no se define a través del tipo de programas computacionales que la integran, sino a través de la clase de información que maneja: información

$4 \quad$ Se conservan las siglas en inglés, CAD (Computer Aided Design), CAM (Computer Aided Mapping), AM-FM (Automate Mapping-Facilities Management) y LIS (Land Information System). 
geográfica o geoinformación. Por lo tanto, todo tipo de aplicación computacional podría ser incluida dentro del concepto de geoinformática, desde las más generales hasta las más específicas, pues todas se relacionan en enlaces de sucesivas vinculaciones que posibilitan la creación de modelos digitales de la realidad.

Como puede verse también en la Figura 4 lo que se ha descrito es la etapa de convergencia en la era geodigital. Esta convergencia que vislumbraba Dobson (1983) hoy se ha cumplido y los SIG evolucionan a través de los Sistemas de Ayuda a la Decisión Espacial (SADE) en un proceso de verticalización (Eastman, 2007). Sin embargo las relaciones Geoinformáticas han superado notoriamente el ámbito acotado de las computadoras personales y sus posibilidades se han ampliado mediante las tecnologías multimedia (como por ejemplo los Atlas interactivos en CD ROM) y su incorporación a la tecnología de Internet a través del llamado GIS On-Line y de toda aplicación que puede realizarse de forma remota en la red.

Actualmente, al mismo tiempo en que se amplían las posibilidades que ofrece el ciberespacio como medio, aparece la Neogeografía, a través de la difusión popularizada de tecnologías en las cuales la componente espacial resulta central y conjuntamente a la disponibilidad de datos geográficos masivos.

Así pues, se vislumbra que estas altas capacidades en la utilización de datos y su procesamiento se dirigirán hacia la realidad virtual, a partir de la cual se experimentarán inmersiones perceptivas en el entorno geográfico digital.

Los primeros pasos en esta dirección se están dando a través de la realidad aumentada, lo cual implica que será necesario realizar un análisis tecnológico de mayor amplitud al relacionarse con estudios psicológicos que aborden la relación entre usuarios y tecnologías, en donde las capacidades comunicacionales de los nuevos medios aparece de forma central (Winter, 2004).

\section{ACTUALIDAD DE LAS TECNOLOGÍAS DE LA INFORMACIÓN GEOGRÁFICA}

Los impactos que las TIG han causado a lo largo de los últimos veinticinco años en el ámbito del análisis espacial han superado con creces lo que las predicciones más optimistas pudieron augurar en los inicios del uso de estas tecnologías, pues al proporcionar recursos técnicos de gran valor que permiten comprender con mayor profundidad los fenómenos que ocurren en la superficie terrestre, han transformado irreversiblemente la manera como hoy en día se abordan los estudios sobre el territorio.

Aumentado progresivamente sus capacidades analíticas, integrando geotecnologías de origen diverso mediante un proceso de convergencia y mejorando su facilidad de uso, las TIG se han convertido en los "nuevos ojos" de las ciencias interesadas en el estudio de la dimensión espacial y han contribuido a llevarlas hasta el siglo XXI, por un lado introduciendo en ellas la tecnificación necesaria para afrontar los retos globales y, por otro lado, expandiendo sus métodos a nuevos ámbitos, científicos y no científicos, en los que eran desconocidos.

De entre todos los cambios causados por las geotecnologías es quizás este último uno de los más significativos pues ha supuesto que las actividades relacionadas con la información geográfica ha- 
yan transcendido los entornos de las computadoras personales y la sobremesa en que se originaron y se hayan extendido circulando ampliamente más allá de ellos.

Este desplazamiento de las TIG y sus aplicaciones fuera de los entornos académicos y profesionales está íntimamente relacionado con la aparición de internet que, actuando a modo de catalizador y multiplicador, ha cambiado radicalmente la manera cómo se han venido utilizando las geotecnologías hasta no hace demasiado tiempo.

La consecuencia más significativa del impacto de la red sobre las TIG, además de facilitar el acceso a un ingente volumen de información geográfica y a un número no menos significativo de herramientas para tratarla, es que gracias a los mecanismos que ella proporciona se ha generado un fenómeno de socialización que ha dado entrada en la escena geográfica global a un nuevo y nutrido grupo de actores, el grueso de la ciudadanía, que de manera muy amplia, rápida y sin prácticamente ningún límite, han empezado a participar de los métodos y procedimientos propios de los expertos geotecnológicos.

Tales individuos o colectivos son conocidos como voluntarios geográficos o neogeógrafos y el proceso resultado de esa participación geográfica, de la socialización geográfica, o lo que algunos llaman wikificación geográfica (Sui, 2008), se ha denominado neogeografía (Turner, 2006).

\section{LA APARICIÓN DE LOS VOLUNTARIOS GEOGRÁFICOS Y LA NEOGEOGRAFÍA}

La aparición del voluntariado geográfico y de la neogeografía es un proceso de largo recorrido, de al menos cincuenta años, que ha acabado cristalizando a inicios del siglo XXI gracias a la aparición y extensión de la red global y al desplazamiento de las geotecnologias hacia esa red, pero también gracias al creciente interés de la ciudadanía por conocer su entorno, a la curiosidad por el "espacio geográfico" que ocupan, su "lugar", entendido como la localización donde llevan a cabo sus actividades cotidianas.

Estos conceptos nucleares de la Geografía y, por lo tanto, totalmente familiares para los especialistas de esta ciencia, han tomado una nueva dimensión, revalorizándose muy significativamente a lo largo del proceso que ha llevado hasta la situación actual.

Los factores que han generado este cambio son múltiples pero destacan especialmente de entre todos ellos tres que cabe considerar fundamentales (Ruiz, 2010b).

En primer lugar el proceso de tecnificación de la Geografía y la Cartografía que a partir de los años 1950 incorporan, a sus métodos analógicos tradicionales de trabajo, desarrollos de automatización basados en el uso computacional. En segundo lugar el desarrollo y progresiva convergencia de diversos componentes de las TIG (sistemas de información geográfica, sistemas de posicionamiento global, sistemas de teledetección y procesamiento de imágenes de satélite, sistemas basados en la localización, estándares geográficos, geoservidores de datos, etc...) y, finalmente la ya mencionada voluntad ciudadana, generalmente desinteresada y altruista, de participar activamente en la definición, generación e, incluso, análisis de la información geográfica. 
Todos estos elementos se han visto propulsados por la red global que ha acelerado notablemente las dos dimensiones fundamentales de la existencia humana: el tiempo, que ha dejado de ser lento para pasar a ser inmediato (todo es "ahora") y el espacio, que ha dejado de ser lejano para ser siempre próximo (todo está "aquí").

La concurrencia simultánea de estas circunstancias ha provocado una verdadera explosión geográfica, esa extensión ya referida de lo territorial hacia la ciudadanía que ahora usa productos cartográficos, herramientas especializadas y datos geográficos de una manera natural. Este hecho les convierte en la práctica en geoconsumidores, por ejemplo cuando utilizan dispositivos móviles, verdaderas baterías de sensores geográficos, y los centenares de aplicaciones de diversa índole orientadas a la localización que están a su alcance en la red y que cualquiera puede usar dondequiera que esté simplemente llevando en su bolsillo un ingenio portátil de este tipo.

El desarrollo tan importante de esta globalización geográfica (Buzai, 2001, 2004) ha comportado la entrada en contacto de los profesionales y los aficionados que en estos momentos comparten un mismo ecosistema con una intensidad nunca vista hasta la actualidad. Ante esta situación, a menudo la comunidad de especialistas se ha mostrado recelosa y ha observado con preocupación los inconvenientes que las acciones de los nuevos actores voluntarios pueden comportar, especialmente en cuanto a la veracidad y la calidad de los datos geográficos que generan.

Pero lo verdaderamente significativo debiera ser dejar de lado las suspicacias para intentar compatibilizar ambos mundos, dotando a los neogeógrafos de los conocimientos necesarios para que utilicen correctamente las nuevas posibilidades que ahora tienen a su alcance y, de esta manera, evitar que la explosión geográfica acabe deteriorando el conocimiento que hemos acumulado durante centenares de años sobre el planeta tierra.

Del mismo modo se tendrían que reconocer las ventajas que supone la entrada en juego de los voluntarios geográficos en cuanto a sus capacidades para actuar con mayor rapidez, flexibilidad y en mayor número que los profesionales (que están sujetos a protocolos, sistematización, procedimientos, etc) para lograr sus metas. El objetivo final ante la situación surgida debería pasar por intentar romper las barreras que separan ambos mundos para aprovechar sinergias y, de este modo, mejorar nuestro saber geográfico global.

\section{EL PAPEL DE LOS VOLUNTARIOS GEOGRÁFICOS}

Los papeles que ejercen los voluntarios geográficos son tremendamente amplios y diversos, pues abarcan un espectro de actividades que van desde el simple uso de la información territorial que se encuentra disponible en la red, hasta la generación de sofisticadas aplicaciones informáticas de carácter geográfico.

Hasta el momento actual los neogeógrafos se han centrado mayoritariamente en el uso de manera pasiva de la información geográfica proporcionada por terceros (observando imágenes en alguno de los múltiples geovisores fotográficos disponibles en la red, usando mapas interactivos de diversa índole, empleando herramientas desarrolladas para calcular distancias, manejando aplicaciones para el uso de los transportes públicos o para localizar servicios o personas, etc). Pero de un tiempo a esta parte cada vez es más frecuente observar como los más avezados empiezan a ir más allá al ser 
capaces de generar su propia información geográfica con la ayuda, por ejemplo, de receptores del sistema de posicionamiento global (GPS).

Así pues, elaboran bases cartográficas a medida para aplicaciones concretas que recogen información que les es de su interés (rutas turísticas, localización de elementos significativos o singulares, etc) e incluso, en la cúspide de la pirámide neogeográfica, es posible encontrar proyectos de generación de cartografía que se asemejan cada vez más a los que realizan los institutos cartográficos nacionales. Tal sería el caso de OpenStreetMap (OSM) un proyecto de voluntariado para la elaboración de un mapa global abierto, creado por usuarios no expertos y en el que cualquier individuo puede participar, cuyo único interés es recopilar información geográfica para ponerla al alcance de cualquier ciudadano que la requiera.

Todas estas acciones tienen en común que se organizan de manera significativamente distinta a como lo hacen las actividades convencionales. Como ya se ha mencionado, en ellas los protagonistas son individuos o colectivos que por voluntad propia deciden actuar en pro de cierta actividad geográfica y ello comporta que tengan un gran dinamismo y evolucionen con gran rapidez.

Suelen ser muy poco rígidas en cuanto a reglas y prácticas, horizontales en cuanto a funcionamiento (de hecho son heterárquicas pues se da la mixicidad, la superposición de funciones, el consenso en la toma de decisiones, etc) y a menudo poco concretas (Goodchild, 2009; Flanagin y Mercer, 2008).

Estas características las hacen especialmente vulnerables básicamente en cuanto a su longevidad pues es muy frecuente que la entrada y salida de participantes en un proyecto acabe diluyéndolo o reorientándolo respecto a sus objetivos originales o, simplemente, lleve a su desaparición.

Pero por otro lado las mismas características pueden suponer ciertas ventajas respecto a estructuras más convencionales, ya que al no hallarse sujetos a formalismos o la rigidez de normas preestablecidas, pueden actuar con mayor rapidez en ciertas situaciones, por ejemplo ante emergencias producidas por desastres naturales, o con mayor intensidad agregando los miembros que sean necesarios a la estructura existente para conseguir sus objetivos.

Aunque los beneficios de este tipo de actividades resultan evidentes, no debe olvidarse que el uso y la generación de información geográfica no es algo trivial, pues de ella pueden depender muchas de las acciones que los seres humanos emprendemos respecto del territorio que ocupamos. Si se piensa, por ejemplo, que la calidad de la información geográfica tiene un papel fundamental en los anteriormente mencionados Sistemas de Ayuda a la Decisión Espacial (SADE), resulta evidente que se debe ser precavido y riguroso a la hora de definir qué papel pueden desempeñar los neogeógrafos y que credibilidad se puede otorgar a los datos que generan.

Una alternativa razonable que permitiría aprovechar de manera conveniente todos estos esfuerzos pudiera ser la de acreditar la información voluntaria estableciendo ciertos procedimientos que, respetando las estructuras y las maneras de funcionar de estos colectivos, asegurasen la confianza en los datos que generan. Algunos aspectos a observar en este sentido podrían ser:

- Analizar el vacío que pueden llenar los voluntarios con el objetivo de proponer ámbitos de actuación. 
- Establecer criterios que ayuden a saber cuando tiene sentido o no usar datos neogeográficos.

- Analizar los riesgos y los beneficios que comportaría la colaboración entre profesionales y voluntarios.

- Definir qué recursos cabe destinar para que la colaboración entre ambos colectivos sea efectiva.

- Observar que restricciones pudiera haber en el nuevo contexto de colaboración, por ejemplo de carácter legal.

- Establecer procedimientos para la verificación y evaluación de los datos generados.

- Consolidar la continuidad de los proyectos que se inicien en el marco de la colaboración, asegurando un suficiente número de participantes y su mantenimiento en el tiempo.

- Someter los trabajos de los voluntarios a ciertos estándares: de calidad, de seguridad, de autoridad, etc.

Para alcanzar estos propósitos se podrían desarrollar protocolos que permitiesen integrar la labor que se realiza en ambos mundos, sumando capacidades para avanzar hacia la convivencia útil entre profesionales y voluntarios por la vía del diálogo, el consenso y la interacción.

Ignorar la labor de los neogeógrafos quizás sería un error, pero también lo sería otorgarle credibilidad sin más. Combinar el conocimiento adquirido a lo largo de los años por el mundo geográfico académico y profesional y el que adquieren a diario los voluntarios debería ser una alternativa a considerar detenidamente.

\section{EL FUTURO DE LAS TIG: EXPECTATIVAS Y RETOS}

El panorama presentado hasta el momento ha permitido constatar como las tecnologías de la información geográfica han traspasado los nichos habituales donde se generaron y permanecieron durante muchos años y se han extendido entre la población, en un movimiento explosivo que ha llegado mucho más lejos de lo que nunca fue capaz de lograr la Geografía académica (Ruiz, 2010a).

Es en este contexto de desplazamiento desde un mundo geográfico analógico y cerrado (la era geoanalógica) a uno digital y abierto de par en par (la era geodigital), que el gran valor que siempre tuvo la Geografía como ciencia para el conocimiento de la superficie terrestre se ha potenciado gracias a las geotecnologías que permiten compartir y comunicar esos saberes con rapidez y eficacia.

Con las TIG actuando a modo de altavoces geográficos, el lenguaje propio de la Geografía se ha convertido en un lenguaje común para la ciudadanía que está adoptándolo con naturalidad. A estas alturas nadie ya se siente extraño observando una ortofotografía o una imagen de satélite, lo cual si se piensa detenidamente es algo extraordinario y fascinante. 
Las geotecnologías, por lo tanto, se están convirtiendo en nuevos medios de comunicación de masas que la población utiliza para compartir e intercambiar información territorial (Sui y Goodchild, 2012).

Esta transformación se lleva a cabo, en primera instancia, en el mundo virtual donde existen herramientas geográficas que permiten recoger las reivindicaciones que los ciudadanos puedan efectuar sobre un determinado territorio y articular entornos para la cooperación y la creatividad colectiva.

En una segunda instancia, si cabe más significativa que la primera, los neogeógrafos están empezando a trasladar el conocimiento geográfico adquirido en los entornos virtuales al mundo real y lo ponen en funcionamiento realizando actuaciones de intervención territorial con el objetivo de recuperar el espacio de su comunidad. Sin que este tipo de acciones sea algo totalmente novedoso, lo cierto es que las circunstancias en que se producen con la existencia de la red, de la información geográfica y las geotecnologías disponibles en internet las han potenciado muy significativamente.

Este contexto conduce, muy probablemente, hacia el desarrollo de una inteligencia geográfica global, desde la tecnosfera hacia la noósfera por la totalidad del sistema mundo y distribuida a través de la misma tecnosfera. Se evoluciona hacia un sistema nervioso planetario, la tierra digital (Craglia et al., 2008, 2012) entendida como una representación tridimensional virtual del planeta con múltiples escalas y temas que permitirá localizar, visualizar y analizar la ingente cantidad de datos geográficos que se están almacenando, por ejemplo a través de los ya citados instrumentos de realidad aumentada, y a partir de ellos actuar sobre el territorio global a medida que nuestra inteligencia espacial aumente.

A este contexto que se expone cabe aún añadir otros elementos que justo en estos momentos se están desarrollando y tomando forma y que apuntan hacia el escenario descrito. Nos referimos a los avances que se están realizando en el campo de la sensorización del entorno mediante el despliegue en el territorio de dispositivos de recolección autónoma de información, que llevan hacia el concepto de web 3.0, o la internet de las cosas, y que están a punto de convertir a objetos inanimados en fuentes de datos geográficos que deberemos aprender a manejar e integrar en nuestros análisis.

Del mismo modo se está avanzando significativamente en la sensorización de los propios seres humanos de manera que estos empiezan a actuar como recolectores ambulantes pasivos e involuntarios de información geográfica a través de las tecnologías móviles ya aludidas con anterioridad, o mediante el uso de dispositivos que pueden estar incorporados en prendas de vestir o simplemente usando las redes sociales que incorporan nuevas funcionalidades espaciales.

Otro ejemplo que parece indicar que se sigue el camino que nos llevará a desarrollar una realidad geográfica virtual lo proporciona el uso intensivo que se viene realizando de los Vehículos Aéreos No Tripulados (VANT, o en inglés Unmanned Aerial Vehicles-UAV o, simplemente, drone) los cuales, cuando se usan en proyectos civiles y de investigación no relacionados con actividades militares, permiten recolectar gran cantidad de información geográfica, de gran calidad, a muy gran escala y con un coste muy reducido si se compara con los sistemas tradicionales de fotografía aérea o fotogrametría. 
Toda esta situación supondrá la generación, por parte de los actores geográficos y cartográficos tradicionales y de los nuevos usuarios de las geotecnologías, de un ingente volumen de datos geográficos recogidos en tiempo real, abiertos, ubicuos, multidimensionales $(3 \mathrm{D}, 4 \mathrm{D})$, heterogéneos, interconectados, en alta definición, que estarán almacenados en la red (computación en la nube), etc. (UN, 2013). Parece ser que la avalancha de información geográfica, los datos geográficos masivos (o el big data geográfico), se encuentra a la vuelta de la esquina y se debe estar preparado para ello.

\section{CONSIDERACIONES FINALES}

La población mundial vive rodeada de las cuatro grandes esferas del mundo. Sin embargo, cada vez más actividades científicas y cotidianas se desarrollan en la Geotecnósfera, la esfera de las tecnologías digitales que incorporan la dimensión espacial.

Los retos que esta novedosa situación plantea son muy importantes. Nuestro objetivo debería centrarse en aprovechar positivamente el curso de los acontecimientos para, de este modo, poder convivir en el nuevo contexto, creando un marco de colaboración efectivo que intente reducir las distancias existentes entre los distintos colectivos que operan en la geografía real, eludiendo los usos indebidos que puedan aparecer con la nueva situación (por ejemplo, para escapar de un indeseable efecto "gran hermano" o de la privatización de la información geográfica recopilada) y compartiendo experiencias y conocimientos para evitar que pasemos de los datos geográficos masivos al naufragio del conocimiento territorial. Actuando de esta manera será posible enfrentar con suficientes fundamentos los retos globales que ya empiezan a emerger ante nosotros.

\section{REFERENCIAS}

Boido, G. 1996. Noticias del Planeta Tierra. Galileo Galilei y la revolución cientifica. AZ. Buenos Aires.

Bunge, M. 1981. La ciencia. Su método y su filosofia. Siglo Veinte. Buenos Aires.

Burrough, P.A.; McDonnell, R.A. 1998. Principles of Geographic Information Systems. Oxford University Press. Oxford.

Buzai, G.D. 2001. Paradigma Geotecnológico, Geografía Global y CiberGeografía, la gran explosión de un universo digital en expansión. GeoFocus, 1:24-48.

Buzai, G.D. 2004. Geografia global. Lugar Editorial. Buenos Aires.

Buzai, G.D. 2008. Consideraciones sobre el rol científico de los Sistemas de Información Geográfica (SIG) a dos décadas de "Pensamientos sobre la Geografía" de Peter Gould. Huellas. 12:265-281.

Buzai, G.D. 2013a. Geografía y pensamiento geográfico. Articulación de enfoques para la investigación aplicada basada en Sistemas de Información Geográfica. Conferencia. Universidad 
Nacional Autónoma de México. 26 de Seriembre.

Buzai, G.D. 2013b. Technological Dependency and the Internet: Latin American Access from Buenos Aires, 2001-2013. Journal of Latin American Geography. 12(3):165-177.

Buzai, G.D.; Baxendale, C.A. 2011. Análisis Socioespacial con Sistemas de Información Geográfica. Tomo 1 - Perspectiva cientifica / temáticas de base raster. Lugar Editorial. Buenos Aires.

Buzai, G.D.; Baxendale, C.A.; Cruz, M.R. 2009. Fases de un proyecto de investigación en estudios de Geografía Aplicada basados en el uso de Sistemas de Información Geográfica. Fronteras. 8(8):31-40.

Buzai, G.D.; Cacace, G. 2013. El concepto de espacio. Si Muove. 5:34-38.

Craglia, M. et al. 2008. Next generation digital earth. A position paper from the Vespucci initiative for the advancement of geographic information systems. International Journal of Spatial Data Infrastructure Research. 3:146-167.

Craglia, M. et al. 2012. Digital Earth 2020: towards the vision for the next decade. International Journal of Digital Earth. 5(11):4-21.

De Rosnay, J. 1977. El Macroscopio. Hacia una visión global. AC. Madrid.

Dobson, J.E. 1983. Automated Geography. Professional Geographer. 35(2):135-143.

Dollfus, O. 1992. Systeme Monde et Systeme Terre. L'Espace Géographique. 21(3):223-229.

Eastman, J.R. 2007. La verticalización de los Sistemas de Información Geográfica. En: Buzai, G.D. (ed.) Memorias de la XI Conferencia Iberoamericana de Sistemas de Información Geográfica. Universidad Nacional de Luján. Luján. pp. 183-195.

Flanagin, A.J.; Metzger, M.J. 2008. The credibility of volunteered Geographic information. Geojournal. 72:137-148.

Focás, B. 2013. En el "cibermapa" el país limita con Estados Unidos, Italia y Francia (Reporte de la investigación de Gustavo Buzai), Perfil, Domingo 28 de junio, pp. 44-45.

García, R. 1997. La Epistemología Genética y la Ciencia Contemporánea. Gedisa. Barcelona.

García, R. 2006. Sistemas Complejos. Gedisa. Barcelona.

Goodchild, M.F. 2009. Neogeography and the nature of geographic expertise. Journal of location based services. 2(2):82-96.

Gould, P. 1987. Pensamientos sobre la Geografía. GeoCrítica. 68:1-39.

Lakatos, I. 1977. La metodología de los programas de investigación científica. Alianza. Madrid. 
Lévy, J. 2012. La definición del geógrafo. Altas de las Mundializaciones. Le Monde Diplomatique. Fundación Mondipló. Valencia. p.24.

Piaget, J.; García, R. 1983. Psicogénesis e historia de la ciencia. Siglo XXI. Madrid.

Piq, P. 2012. La definición del antropólgo. Altas de las Mundializaciones. Le Monde Diplomatique. Fundación Mondipló. Valencia. pp. 21-22.

Rand, A. 2011. Introducción a la Epistemología Objetivista. El Grito Sagrado. Buenos Aires.

Ruiz, E. 2010a. El impacto de las tecnologías de la información geográfica en la cartografía y la geografía: reflexiones sobre 20 años de SIG. En: Buzai, G.D. (Ed.) Geografia y sistemas de información geográfica. Aspectos conceptuales y aplicaciones. GESIG-PROEG. Luján, pp. 51-64.

Ruiz, E. 2010b. Consideraciones acerca de la explosión geográfica: Geografía colaborativa e información geográfica voluntaria acreditada. GeoFocus (Artículos), 10:280-298.

Sagan, C. 1980. Cosmos. The Scott Meredith Library Agency. New York.

Sagan, C. 1995. ¿Podemos conocer el universo? En: Gardner, M. (ed) El escarabajo sagrado (I). Salvat. Barcelona. pp. 115-121.

Soros, G. 2010. The Soros Lectures at the Central European University. Public Affairs. New York.

Stotman, 1999. Conferencia. Congreso sobre la enseñanza de la Geografía frente a un mundo en cambio. Universidad Nacional de Cuyo. 19 al 24 de Abril. Mendoza.

Sui, D. 2008. The wikification of GIS and its consequences: Or Angelina Jolie's new tattoo and the future of GIS. Computers, Environment and Urban Systems. 32(1):1-5

Sui, D.; Goodchild, M. 2011. The convergence of GIS and social media: challenges for GIscience. International Journal of Geographical Information Science. 25:11. 1737-1748.

Turner, A.J. 2006. Introduction to Neogeography. O’Reilly Media. Sebastopol.

United Nations. 2013. Future trends in geospatial information management: the five to ten years vision. United Nations Initiative on Global Geospatial Information Management. Disponible en

http://ggim.un.org/docs/meetings/3rd\%20UNCE/UN-GGIM-Future-trends.pdf. 31 de julio de 2013.

Von Bertalanffy, L. 1968. General Systems Theory: Foundations, Developmen, Applications. George Brazillier. New York.

Winter, S. 2004. Communications about space. Transactions in GIS. 8(3):291-296. 